\title{
Residual Vibration Reduction in Mechanical Systems: A Time-domain Approach
}

\author{
Joaquim M. Veciana ${ }^{1, \#}$, Salvador Cardona ${ }^{1}$ \\ 1 Department of Mechanical Engineering, Polytechnic University of Catalonia, ETSEIB, Diagonal 647, Barcelona, Spain, 08028 \\ \# Corresponding Author / E-mail: joaquim.maria.veciana@upc.edu, TEL: +34-93-4016718, FAX: +34-93-4015813
}

KEYWORDS : Residual vibration; Residual response; Vibration control; Command shaping; Input shaping; Forcing function

This paper presents a time-domain technique to generate command inputs for the reduction of residual vibrations in mechanical systems. The technique is based on the generation of motion profiles with zero-crossing points in their frequency spectra at the system resonances in the same way as the zero-vibration (ZV) command generation methods. By including an appropriate negative exponential time function, which contains the system natural frequency and the damping ratio, the resultant input can be applied to damped systems with a result of zero residual vibration. The signals synthesized can be used as a generic physical magnitude in vibratory systems. However, they were particularized for mechanical systems and engineering metrics were used. Compared to other ZV methods, for a short duration input range, the signals obtained have better performance in terms of minimum-acceleration-switch shape which is translated to a smoother motion profile. The development includes the application to single-mode and multiple-mode mechanical systems and the possibility of handling two functional requirements or constraints in the desired application. The analyzed systems are those that can be modeled as discrete linear ones with several vibratory degrees of freedom and can be described with constant parameter motion equations. Experimental results show the efficacy of the method developed with its application to a damped pendulum test platform.

\section{Introduction}

A vibratory or oscillatory behavior, functionally noticeable, is commonly found in mechanical systems due to the inertia and stiffness of the components. During their normal operation, transient excitations may occur because of external forces or the movement of internal inertias, which normally raise this vibratory reaction. The intended function of the mechanism may be compromised because the vibrations generated usually remain in the system after the end of the transient excitation. Motion control has been one of the main areas of focus for the development of residual vibration reduction. Much work has been carried out with methods based on command generation techniques applied to discrete linear systems ${ }^{1,2}$. Most of them were developed by focusing on the optimization of the transient time. However, in the short duration time range, between 1 and 1.5 periods of the system natural frequency, the resultant shapes of the generated input functions are not optimal in terms of minimum-acceleration-switch condition, with peak values that are far from the average intended value. Therefore, the amplitude of the system response is usually noticeable, although it depends on the unshaped command used.

Assume that the inputs of the Figure 1 are velocity profiles $\dot{y}(t)$ that define a point-to-point maneuver. The number of acceleration switches is one (optimal condition), two and three for the indicated curves. In this context, unwanted dynamics, such as high order modes, can be excited by transmission backlashes, mechanical plays or electronic hysteresis, when the input function alternates between positive and negative values of acceleration. Furthermore, such condition can also imply a premature wear of contact surfaces and fatigue of mechanical parts.

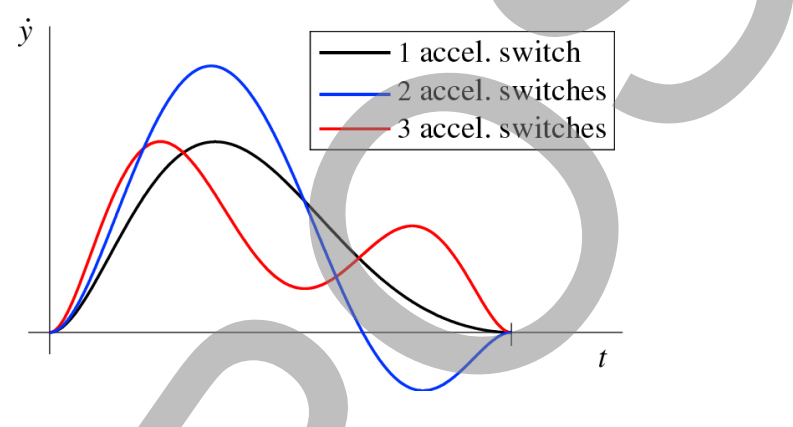

Fig. 1 Point-to-point velocity profiles with different acceleration-switch condition

In this way, the signal generation based on the definition of transient excitations through trigonometric series expansion of functions present rippled profiles, because the series used were truncated and the terms related with the system natural frequency were not included ${ }^{3-5}$.

The zero-vibration (ZV) input shapers ${ }^{6,7}$ and their extensions zero-vibration and derivative (ZVD) and extra insensitive (EI) shapers, which provide robustness against variation of system parameters, were obtained by convolution of an unshaped signal by a sequence of impulses. These methods do not, in general, satisfy the minimum-acceleration-switch condition. Other approaches of this technique included: i) phasorial diagrams to reduce numerical calculations ${ }^{8,9}$; ii) negative impulses to reduce the transient time ${ }^{10}$; iii) an optimal trade-off between performance, 
measured in terms of residual vibration and speed, and robustness ${ }^{11}$; iv) the cancellation of a so-called pseudo-mode with lower frequency than any of the system component modes ${ }^{12}, \mathrm{v}$ ) the reduction of the system response during the transient ${ }^{13,14}$. All these methods obtained the desired command input through the impulse shaping as well, presenting a non minimum-acceleration-switch condition.

Conventional command filtering was studied to eliminate the frequency content at the system resonance from the input ${ }^{15}$. In this case, the resultant signals usually show a minimum-acceleration-switch condition in all time ranges. However this technique is not considered an analytically proven ZV method: Although it is demonstrated that reducing the frequency content at the system resonance is equivalent to reducing the residual vibration ${ }^{16}$, the lower effectiveness of the filtering methods can be justified because this statement is only valid for undamped systems. Therefore, those methods do not give a total residual vibration reduction in damped systems.

Methods involving discrete filters were also developed. The zero-placement technique ${ }^{17}$ was extended to the discrete domain by constructing the impulse sequences in the z-plane ${ }^{18}$. The Optimal Arbitrary Time-delay Filter (OATF) ${ }^{19}$, where its time-domain representation is a set of positive and negative impulses with variable time-delay. In these techniques, the desired signal is generated again by convolving an unshaped command with a sequence of impulses. In the time range indicated, the non minimum-acceleration-switch condition of the resultant inputs is noticeable as well.

The main objective of the present work is to provide input functions with analytically proven null residual vibration (ZV), which present a minimum-acceleration-switch condition during the transient, compared to other literature methods. The main contribution is the development of a zero vibration method for $n$ degree-of-freedom damped linear systems to generate those command inputs. It involves the time-domain rescaling of an arbitrary base function to locate the zero-crossing points of its frequency spectrum at the system damped frequencies, and the later product of this base signal with an appropriate negative exponential time function. Unlike the previously presented input shaping methods, this approach cannot be implemented in real time. The entire command must be computed before the motion is initiated.

The rest of this paper is organized as follows. In Section 2, the mathematical analysis is introduced, which derives in the frequency domain the necessary and sufficient condition that an input should meet to eliminate residual vibrations in a damped system. The design method and two strategies to fix two functional constraints are explained. In section 3, the extension to multiple-mode systems is detailed and it is proven that the time-derivatives or integrals of the formulated profiles are also useful to reduce residual vibrations. In Section 4, a comparative simulation with the main ZV techniques is presented ${ }^{6,10,19}$. Next, in Section 5, some experimental results are shown by using a pendulum-carriage test bed that is essentially a crane. In such systems, the input shaping has been commonly applied ${ }^{20-23}$. Finally, in Section 6 , conclusions are drawn, pointing out the benefits of the method developed.

\section{Input Design Method}

The development carried out in this paper can be extended to any vibratory system assuming that $y(t)$ and $x(t)$ are time functions of physical magnitudes (force, position, velocity, acceleration, voltage, current...) which can be considered as the input and the response of this system, respectively. However, to clarify the development, it is particularized to the mechanical system in Figure 2, assuming that the input is the absolute coordinate of the base $y$, and the output is the relative coordinate of the mass $x$.

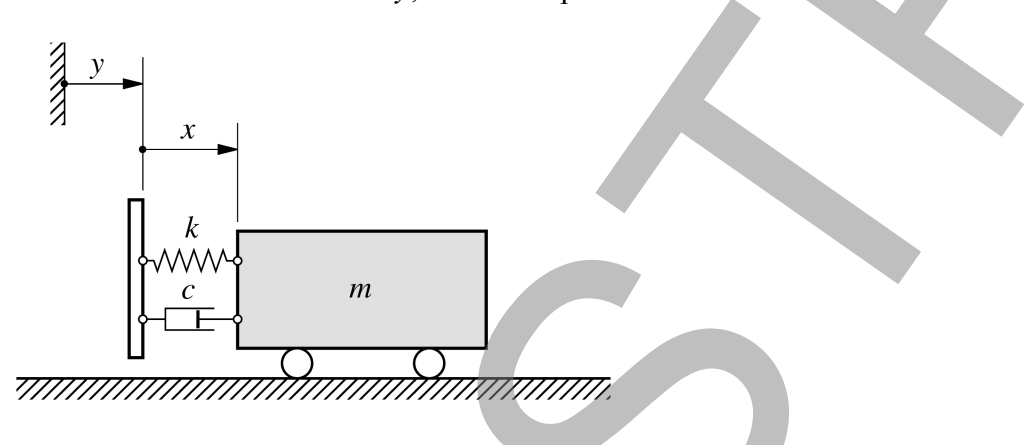

Fig. 2 Generic single-mode discrete linear system

\subsection{Necessary and sufficient condition}

The necessary and sufficient condition for zero residual vibration is that the Laplace transform (LT) of the time-bounded control input $y(t)$ has zero magnitude at the system poles. This condition was proven previously by other authors ${ }^{12,17,24}$. In this section, we introduce the necessary and sufficient condition for zero residual vibration, but explained through the Fourier transform (FT) in which is based the new method developed.

The motion equation of the system of the Figure 2 is given by

$$
m \ddot{x}+c \dot{x}+k x=-m \ddot{y}(t)
$$

this expression can be rewritten as

$$
\ddot{x}+2 \zeta\left(2 \pi f_{0}\right) \dot{x}+\left(2 \pi f_{0}\right)^{2} x=-\ddot{y}(t)
$$

where $f_{0}=\sqrt{k / m} /(2 \pi)$ is the system natural frequency and $\zeta=c /(2 \sqrt{m k})$ is the damping ratio.

Assuming a vibratory behavior $(\zeta<1)$, the system poles are $s_{1,2}=-\zeta 2 \pi f_{0} \pm \mathrm{j} 2 \pi f_{\mathrm{d}}$ where $f_{\mathrm{d}}=f_{0} \sqrt{1-\zeta^{2}}$ is the oscillation frequency or damped 
frequency. Therefore, the stated necessary and sufficient condition yields

$$
Y\left(s_{1,2}\right)=\int_{t_{0}}^{t_{\mathrm{f}}} y(t) \mathrm{e}^{-\left(-\zeta 2 \pi f_{0} \pm \mathrm{j} 2 \pi f_{\mathrm{d}}\right) t} \mathrm{~d} t=0
$$

To switch from the necessary and sufficient condition described through the LT to the one described through the FT, we introduce $g(t)=y(t) \mathrm{e}^{\zeta 2 \pi f_{0} t}$ in (3) and the resultant expression (4) is compared to the development of the FT of $g(t)$ evaluated at $f_{\mathrm{d}}$ in expression (5).

$$
\begin{aligned}
& Y\left(s_{1,2}\right)=\int_{t_{0}}^{t_{\mathrm{f}}} g(t) \mathrm{e}^{\mp \mathrm{j} 2 \pi f_{\mathrm{d}} t} \mathrm{~d} t= \\
& \int_{t_{0}}^{t_{\mathrm{f}}} g(t) \cos \left(2 \pi f_{\mathrm{d}} t\right) \mathrm{d} t \mp \mathrm{j} \int_{t_{0}}^{t_{\mathrm{f}}} g(t) \sin \left(2 \pi f_{\mathrm{d}} t\right) \mathrm{d} t=0 \\
& \left.\operatorname{FT}[g(t)]\right|_{f_{\mathrm{d}}}=G\left(f_{\mathrm{d}}\right)= \\
& \int_{t_{0}}^{t_{\mathrm{f}}} g(t) \cos \left(2 \pi f_{\mathrm{d}} t\right) \mathrm{d} t-\mathrm{j} \int_{t_{0}}^{t_{\mathrm{f}}} g(t) \cos \left(2 \pi f_{\mathrm{d}} t\right) \mathrm{d} t
\end{aligned}
$$

The integrals of Expression (4) and (5) are the same. Therefore, to say that the LT of the time-bounded control input $y(t)$ has zero magnitude at the system poles is equivalent to say that the Fourier transform of the function $y(t) \mathrm{e}^{\zeta 2 \pi f_{0} t}$ has zero magnitude at the oscillation frequency $f_{\mathrm{d}}$.

\subsection{Design method}

Figure 3 shows the flow diagram of the proposed design method. The extension to multiple mode systems is explained in 3.2.

The command input obtained is described by the Expression (6), where $u_{\mathrm{b}}(t)$ is a rescaled base transient function, which contains any zerocrossing point located at $f_{\mathrm{d}}$ in its frequency spectrum, and $A$ is a constant amplitude scale factor to be determined to accomplish one functional requirement.

$$
y(t)=A u_{\mathrm{b}}(t) \mathrm{e}^{-\zeta 2 \pi f_{0} t}
$$

Example: Assume that an acceleration input $\ddot{y}(t)$ is desired and a rectangular pulse, defined between $t_{0}=0$ and $t_{\mathrm{f}}$, is selected as the base function. If the duration of the rectangular pulse is rescaled by fixing $t_{\mathrm{f}}$ at $1 / f_{\mathrm{d}}$, the magnitude of its frequency spectrum is given by Expression (7) and is null at $f_{\mathrm{d}}, 2 f_{\mathrm{d}}, 3 f_{\mathrm{d}}, \ldots$, .

$$
\left|U_{\mathrm{b}}(f)\right|=A\left|\frac{\sin \left(2 \pi f / f_{\mathrm{d}}\right)+\mathrm{j}\left[\cos \left(2 \pi f / f_{\mathrm{d}}\right)-1\right]}{2 \pi f}\right|
$$

By multiplying the rescaled pulse by $A \mathrm{e}^{-\zeta 2 \pi f_{0} t}$, we obtain $\ddot{y}(t)$, which final shape is shown in Figure 4 .

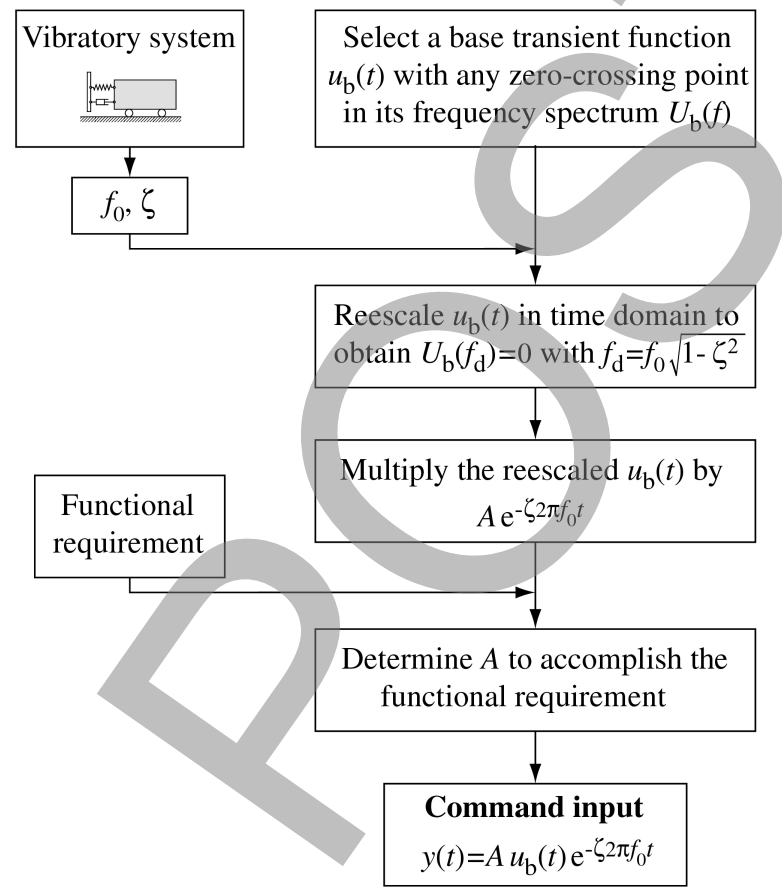

Fig. 3 Flow diagram of the design method for a single-mode system 


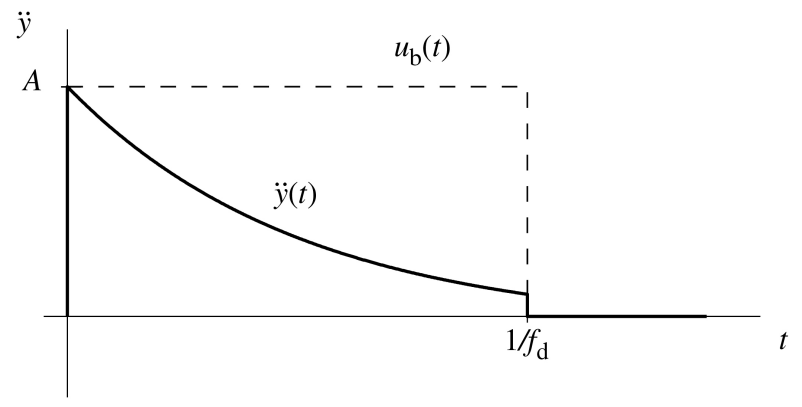

Fig. 4 Example of the input $\ddot{y}(t)$, the result of the product of a rescaled pulse $u \mathrm{~b}(t)$ and an exponential time-function with an exponent $-\zeta 2 \pi f_{0} \mathrm{t}$

The method described requires base functions $u_{\mathrm{b}}(t)$ with zero-crossing points in their frequency spectra. The use of symmetric transient signals is recommended because most of them present zero content at some determined frequencies. In the case of a real even base function $u \mathrm{~b}(t)$, its FT is also real and even and is given by next expression.

$$
\begin{aligned}
& U_{\mathrm{b}}(f)=\mathrm{FT}\left[u_{\mathrm{b}}(t)\right]= \\
& \int_{-\infty}^{+\infty} u_{\mathrm{b}}(t) \cos (2 \pi f t) \mathrm{d} t \quad \text { for } u_{\mathrm{b}}(t)=u_{\mathrm{b}}(-t) \forall t
\end{aligned}
$$

The frequency content is null at $f_{\mathrm{d}}$ when $\left|U_{\mathrm{b}}\left(f_{\mathrm{d}}\right)\right|=0$. An analogous reasoning can be followed when the signal is odd. Nevertheless, the null frequency content at $f_{\mathrm{d}}$ for a non-symmetric function can be described by

$$
\left|U_{\mathrm{b}}\left(f_{\mathrm{d}}\right)\right|=\left|\int_{-\infty}^{+\infty} u_{\mathrm{b}}(t)\left(\cos \left(2 \pi f_{\mathrm{d}} t\right)-\mathrm{j} \sin \left(2 \pi f_{\mathrm{d}} t\right)\right) \mathrm{d} t\right|=0
$$

which implies

$$
\begin{aligned}
& \int_{-\infty}^{+\infty} u_{\mathrm{b}}(t) \cos \left(2 \pi f_{\mathrm{d}} t\right) \mathrm{d} t=0 \\
& \int_{-\infty}^{+\infty} u_{\mathrm{b}}(t) \sin \left(2 \pi f_{\mathrm{d}} t\right) \mathrm{d} t=0
\end{aligned}
$$

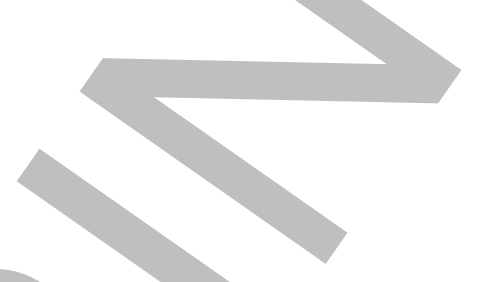

In this case, the null condition is more restricting because the real and imaginary terms must be zero at the same time. Although classical symmetric functions like pulse, trapezium, versine, double harmonic, etcetera have zero-crossing points within their frequency spectra, symmetry is not a sufficient condition to have this property because there are some unusual symmetric functions that do not present this condition.

\subsection{Fixing two functional constraints}

In some motion control applications, the input functions should be capable of achieving two functional requirements or constraints at the same time, such as transient duration, distance to move, velocity increment, etc. In a general case, there is a transient movement of an inertia when a finite variation of one of its kinematic magnitudes (acceleration, velocity or position) occurs in a finite time and, out of this time, this magnitude remains constant. According to this statement, it seems logical that the motion profile includes the finite variation of the motion magnitude (first functional requirement) in a fixed time (second functional requirement). Although the second functional requirement is usually the duration of the command input, in some applications the displacement is used as well.

\section{1- Command generation:}

To handle two constraints, two types of inputs are proposed. The resultant shapes can be significantly different between them, depending on the functions used.

Input i)

$$
y(t)=A\left[u_{\mathrm{b}}(t) \otimes u_{\mathrm{a}}(t)\right] \mathrm{e}^{-\zeta 2 \pi f_{0} t}
$$

where $u_{\mathrm{a}}(t)$ has a duration $t_{\mathrm{a}}$ and is the so-called unshaped command or function. The convolution theorem of the FT states that, under suitable conditions, FT[ $\left.u_{\mathrm{b}}(t) \otimes u_{\mathrm{a}}(t)\right]=U_{\mathrm{b}}(f) \cdot U_{\mathrm{a}}(f)$. If the base function $u \mathrm{~b}(t)$ has zero-crossing points in its frequency spectrum, $u_{\mathrm{b}}(t) \otimes u_{\mathrm{a}}(t)$ has them as well. Therefore $y(t)$ follows the scheme described in the expression (6) as well.

The base transient function $u \mathrm{~b}(t)$ is described in section 2.2. The unshaped function $u_{\mathrm{a}}(t)$ should be a positive function, defined in a finite time range, with a minimum-switch-acceleration condition. Functions that can be used as unshaped commands are, for example, versine, double harmonic or trapezium. 
Input ii)

$$
\ddot{y}(t)=A\left[u_{\mathrm{b}}(t) \mathrm{e}^{-\zeta 2 \pi f_{0} t}\right] \otimes u_{\mathrm{a}}(t)
$$

where $u_{\mathrm{a}}(t)$ is an unshaped function like the one defined in the former case. Suppose that $A u_{\mathrm{b}}(t) \mathrm{e}^{-\zeta 2 \pi f_{0} t}$ is an input function designed following Expression (6) and $x_{1}(t)$ is the system response to this input. The oscillatory term of $x_{1}(t)$ has the same duration of the input because the input has been designed to avoid residual vibrations. If $h(t)$ is the impulse response of the system, $x_{1}(t)$ can be described by $x_{1}(t)=A\left[u_{\mathrm{b}}(t) \mathrm{e}^{-\zeta 2 \pi f_{0} t}\right] \otimes h(t)$. If $A u_{\mathrm{b}}(t) \mathrm{e}^{-\zeta 2 \pi f_{0} t}$ is now convolved with a function $u_{\mathrm{a}}(t)$ with a duration $t_{\mathrm{a}}$, the system response $x_{2}(t)$ can be obtained by

$$
x_{2}(t)=\left(A\left[u_{\mathrm{b}}(t) \mathrm{e}^{-\zeta 2 \pi f_{0} t}\right] \otimes u_{\mathrm{a}}(t)\right) \otimes h(t)
$$

Taking advantage of the associative and commutative properties of the convolution integral, $x_{2}(t)$ can be rewritten as

$$
x_{2}(t)=\left(A\left[u_{\mathrm{b}}(t) \mathrm{e}^{-\zeta 2 \pi f_{0} t}\right] \otimes h(t)\right) \otimes u_{\mathrm{a}}(t)=x_{1}(t) \otimes u_{\mathrm{a}}(t)
$$

Therefore, the duration of the oscillation term of $x_{2}(t)$ is the same as the input, which is the duration of the oscillatory term of $x_{1}(t)$ added to $t_{\mathrm{a}}$ and, hence, there is no residual vibration as well.

\section{2- Algorithms:}

The command amplitude $A$ and the duration of the unshaped function $t_{\mathrm{a}}$ are two parameters to be set to handle the desired constraints. The algorithms presented below describe how to calculate these parameters, considering the two standard cases indicated.

i) Generation of a velocity command $\dot{y}(t)$ : Consider that the input is defined between $t_{0}=0$ and $t_{\mathrm{f}}$. Assume that $t_{\mathrm{f}}$ and the displacement $\Delta y$ are the constraints to be fixed. Figure 5 presents the flow diagram to set the parameters $A$ and $t$ a to achieve those requirements.

ii) Generation of an acceleration command $\ddot{y}(t)$ : Consider again that the input is defined between $t_{0}=0$ and $t_{\mathrm{f}}$, and now, the velocity increment $\Delta \dot{y}$ and the displacement $\Delta y$ are the constraints to be fixed. Figure 6 presents the flow diagram to set the parameters $A$ and $t$ a, to achieve those requirements. The implicit system referred in this figure is given by the expressions (15), (16) and (17).

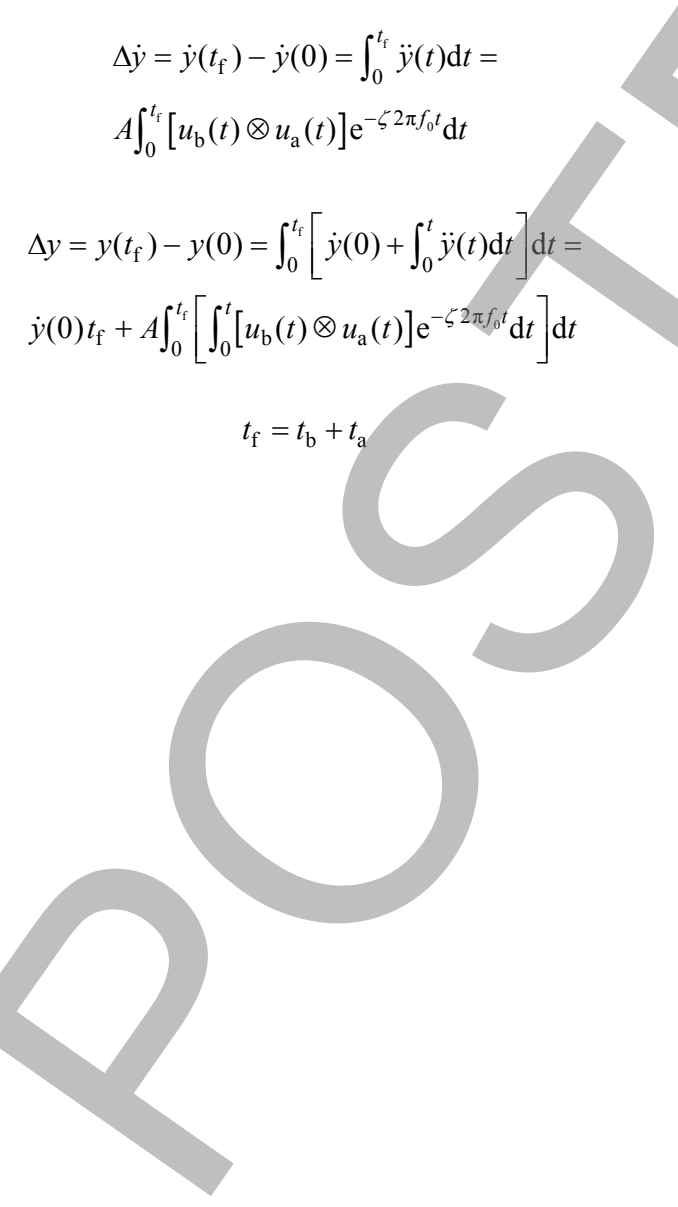




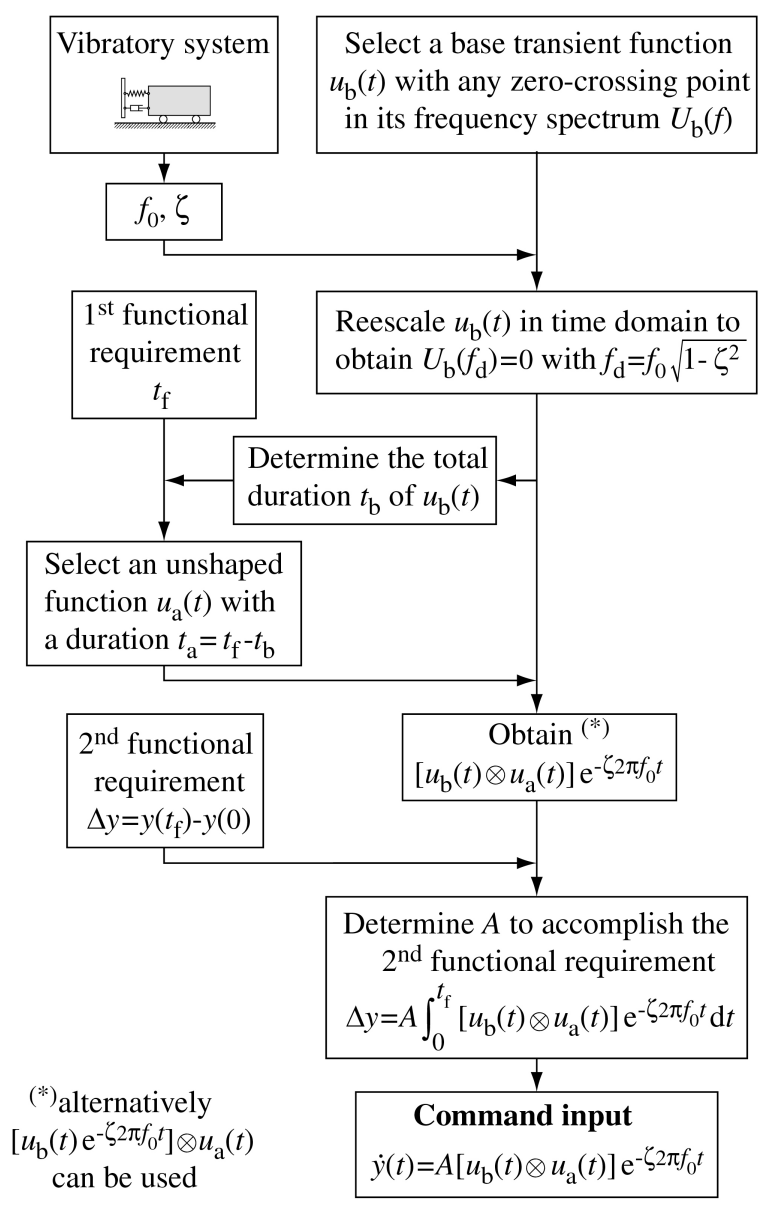

Fig. 5 Flow diagram to generate a velocity command $\dot{y}(t)$ when $t_{\mathrm{f}}$ and $\Delta y$ are functional requirements

Although it is it is possible to include more than two functional constraints in a command by including unknown parameters in the unshaped function $u_{\mathrm{a}}(t)$, the influence on the shape of the resultant profile is very small for short duration motion profiles. Therefore, to find these unknowns for a set of more than two constraints will not be possible in many cases.

Example: An acceleration command $\ddot{y}(t)$ must be designed with the overall time $t_{\mathrm{f}}$ and the velocity increment $\Delta \dot{y}$ as functional constraints. Thus, the flow diagram of the Figure 5 can be used because it is an analogous case. Assume that $u \mathrm{~b}(t)$ is a pulse with a duration $t_{\mathrm{b}}=1 / f_{\mathrm{d}}$. A versine function is selected as unshaped function $u_{\mathrm{a}}(t)$ The parameters $A$ and $t \mathrm{a}$ are given by

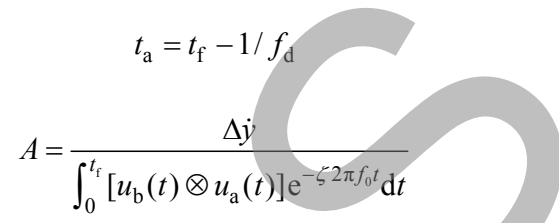

Figure 7 shows the resultant function $\ddot{y}(t)$ where the final input is obtained by multiplying the exponential time function indicated by the result of the convolution $u_{\mathrm{b}}(t) \otimes u_{\mathrm{a}}(t)$. The same figure shows the function obtained by scaling the unshaped function alone. 


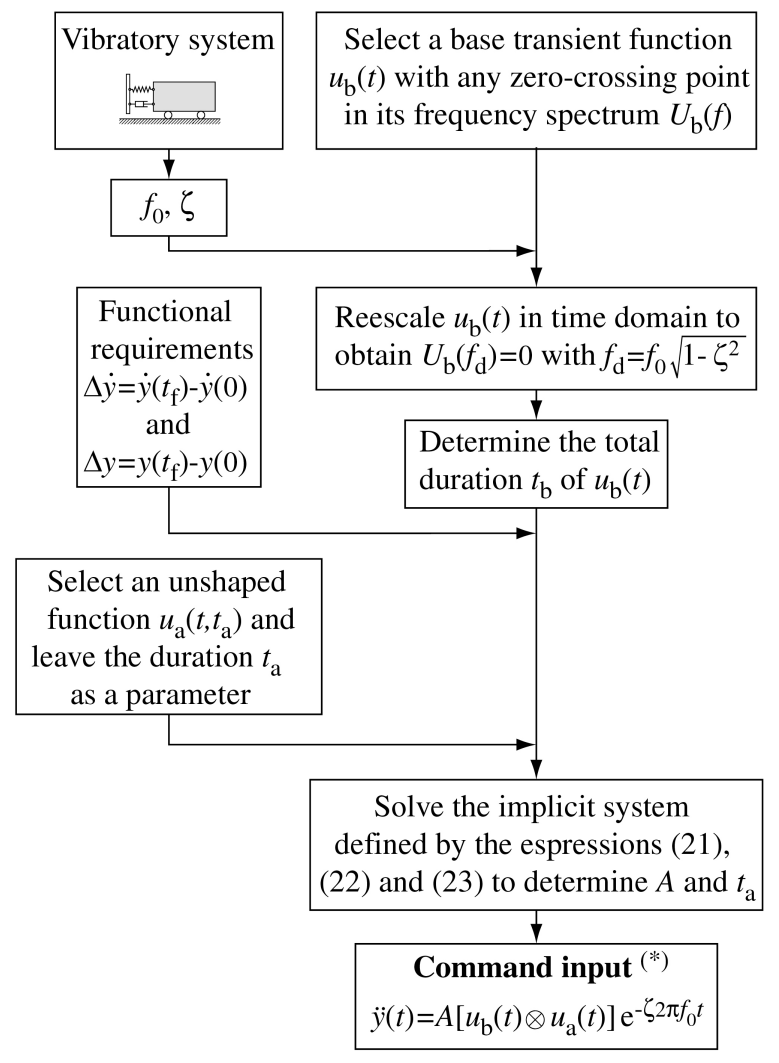

(*)alternatively $\ddot{y}(t)=A\left[u_{\mathrm{b}}(t) \mathrm{e}^{-\zeta 2 \pi f_{0} t}\right] \otimes u_{\mathrm{a}}(t)$ can be used

Fig. 6 Flow diagram to generate an acceleration command $\ddot{y}(t)$ when $\Delta \dot{y}$ and $\Delta y$ are functional requirements

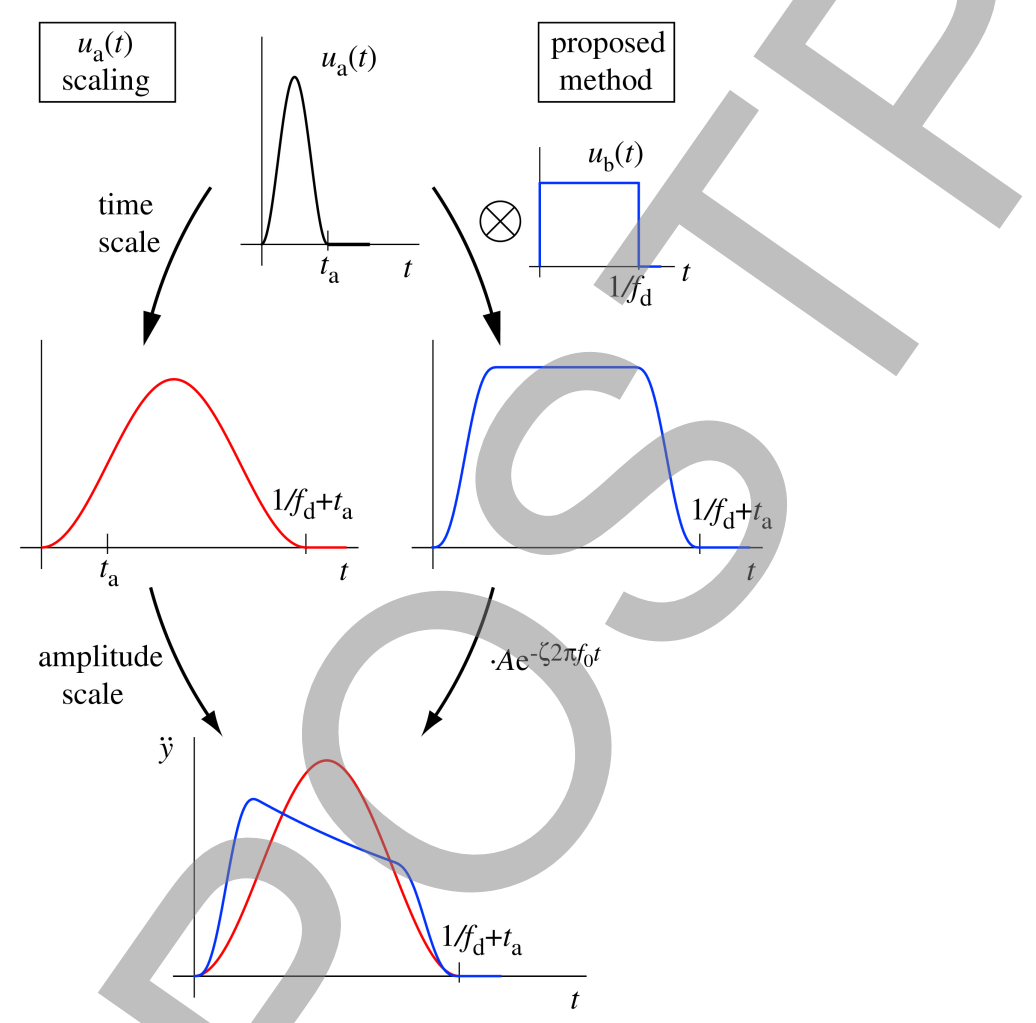

Fig. 7 Fixing two functional constraints with a versine as an unshaped function $u_{\mathrm{a}}(t)$ 


\section{Extensions of the method}

\subsection{Using the derivative or integral to obtain a new input function}

The derivatives or integrals of the functions obtained with the proposed method also satisfy the condition of null residual vibrations. The time derivative of Expression (6) is given by

$$
\dot{y}(t)=A \dot{u}_{\mathrm{b}}(t) \mathrm{e}^{-\zeta 2 \pi f_{0} t}-A \zeta 2 \pi f_{0} u_{\mathrm{b}}(t) \mathrm{e}^{-\zeta 2 \pi f_{0} t}
$$

The second addend of the right term of Expression (20) is the original $y(t)$ multiplied by the constant $\zeta 2 \pi f_{0}$ and, hence, this term does not excite any residual vibration. The first addend contains the derivative of the base function $u \mathrm{~b}(t)$. The properties of the FT state that if $U_{\mathrm{b}}(f)=\mathrm{FT}\left[u_{\mathrm{b}}(t)\right]$ then FT $\left[\mathrm{d} u_{\mathrm{b}}(t) / \mathrm{d} t\right]=\mathrm{j} 2 \pi f U_{\mathrm{b}}(f)$ (provided that $\mathrm{d} u_{\mathrm{b}}(t) / \mathrm{d} t$ exists). Therefore, the points where the frequency content is null in $u \mathrm{~b}(t)$ remain invariable in $\mathrm{d} u_{\mathrm{b}}(t) / \mathrm{d} t$. Thus, the first term of this expression does not excite residual vibrations either. Analogous reasoning can be followed with the integrals of the input defined by Expression (6).

\subsection{Multiple-mode systems}

To cancel multiple mode vibration, several single mode profiles are convolved following the procedure stated by other authors ${ }^{7,25}$. The development described below introduces a time-domain approach.

For a damped $n$-mode linear system with vibratory behavior, according to the modal decomposition theory, the $i$-th coordinate output $x_{i}(t)$ can be described by a sum of weighted and phase-adjusted responses of $n$ single-mode systems, which characterize the natural modes. The oscillatory term of an impulse response $h_{\mathrm{o} i}(t)$ for the coordinate $x_{i}$, assuming a displacement output, is given by the form

$$
h_{\mathrm{o} i}(t)=\sum_{k=1}^{n} C_{i k} \mathrm{e}^{-\zeta_{k} 2 \pi f_{0_{k}} t} \sin \left(2 \pi f_{\mathrm{d}_{k}} t+\varphi_{i k}\right)=\sum_{k=1}^{n} q_{i k}(t)
$$

where $C_{i k}$ and $\varphi_{i k}$ are constants that depend on the system parameters and $f_{0 k}, f_{\mathrm{d} k}$ and $\zeta_{k}$ are, respectively, the natural frequency, the oscillation frequency and the damping ratio of the mode $k$-th.

By using the convolution integral, the function that describes the oscillatory term of the displacement for the $i$-th coordinate $x_{i}(t)$, and for a given input $y(t)$ defined between $t_{0}$ and $t_{\mathrm{f}}$, is

$$
\begin{aligned}
& x_{i}(t)=\int_{t_{0}}^{t} h_{\mathrm{o} i}(t-\tau) y(\tau) \mathrm{d} \tau=\int_{t_{0}}^{t}\left[\sum_{k=1}^{n} q_{i k}(t-\tau)\right] y(\tau) \mathrm{d} \tau= \\
& \sum_{k=1}^{n} \int_{t_{0}}^{t} q_{i k}(t-\tau) y(\tau) \mathrm{d} \tau=\sum_{k=1}^{n}\left[q_{i k}(t) \otimes y(t)\right] \quad i=1, \ldots, n
\end{aligned}
$$

Suppose that for every $k$-th natural mode a transient signal $y_{k}(t)$ is defined following the method explained for single-mode systems. The approach proposed in this study for multiple-mode systems is to construct the input $y(t)$ by convolving the $n$ functions $y_{k}(t)$ :

$$
y(t)=y_{1}(t) \otimes y_{2}(t) \otimes \ldots \otimes y_{k}(t) \otimes \ldots \otimes y_{n}(t)
$$

To prove that the input $y(t)$ does not excite any residual vibration in any natural mode, it is sufficient to demonstrate that the oscillatory term of any coordinate output $x_{i}(t)$ has the same duration as the input $y(t)$. Substituting Expression (23) into (22) gives

$$
\begin{aligned}
& x_{i}(t)=\sum_{k=1}^{n}\left[q_{i k}(t) \otimes y(t)\right]= \\
& \sum_{k=1}^{n}\left[q_{i k}(t) \otimes\left(y_{1}(t) \otimes y_{2}(t) \otimes \ldots \otimes y_{k}(t) \otimes \ldots \otimes y_{n}(t)\right)\right]= \\
& \sum_{k=1}^{n}\left[\left(q_{i k}(t) \otimes y_{k}(t)\right) \otimes\left(y_{1}(t) \otimes y_{2}(t) \otimes \ldots \otimes y_{n}(t)\right)\right] \\
& i=1, \ldots, n
\end{aligned}
$$

The resultant signal given by $q_{i k}(t) \otimes y_{k}(t)$ has the same duration as $y_{k}(t)$, because $y_{k}(t)$ has been designed to cancel the residual vibrations of the $k$-th natural mode. Thus, the duration of the addend $k$-th $(k=1, \ldots, n)$ of Expression $(24)$ is the sum of the durations of $y_{1}(t), y_{2}(t), \ldots, y_{k}(t), \ldots y_{n}(t)$, which is equal to the total duration of $y(t)$. Therefore, the $i$-th oscillatory response of $x_{i}(t)$ has the same duration as the input $y(t)$, which means that there is no residual vibration.

Figure 8 shows an example of an input generated to avoid residual vibrations in a three-mode system. A rectangular pulse is the base function used in this example. The individual functions $y_{k}(t)$ designed for each mode according to this pattern are given by Expression (25). 

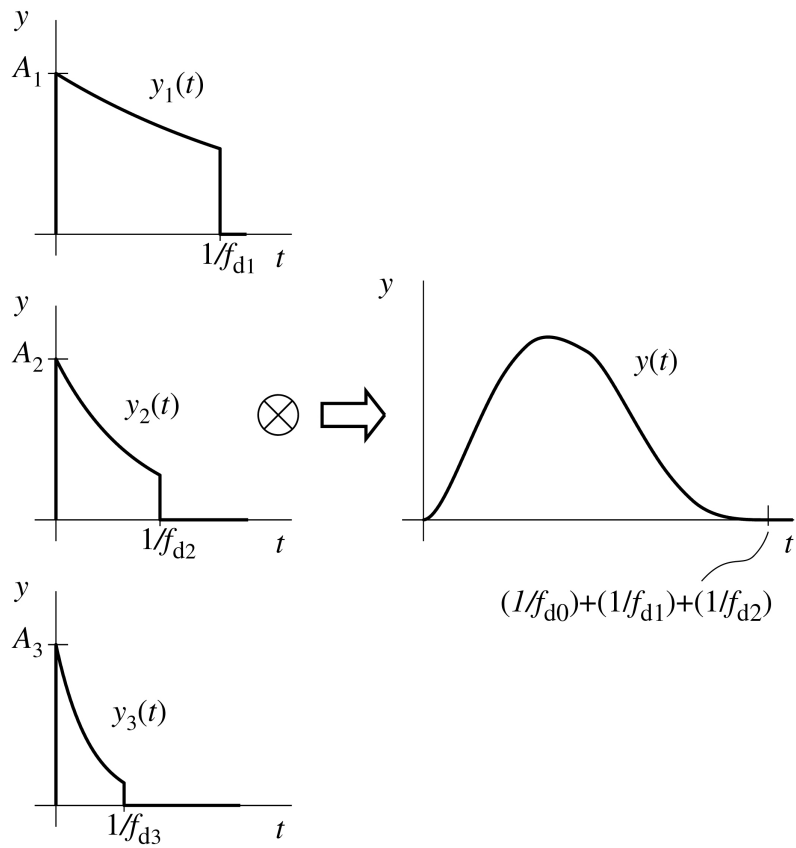

Fig. 8 Input generated to avoid residual vibrations in a three-mode system

$$
\begin{aligned}
& y_{k}(t)=A_{k} \mathrm{e}^{-\zeta_{k} 2 \pi f_{0 k} t} \quad 0 \leq t<1 / f_{\mathrm{d} k} \quad k=1, \ldots, 3 \\
& y_{k}(t)=0 \quad\left\{\begin{array}{l}
t<0 \\
t \geq 1 / f_{\mathrm{d} k}
\end{array}\right.
\end{aligned}
$$

The final shape of the input $y(t)$, obtained by convolving $y_{k}(t) k=1, \ldots, 3$, is also shown in the figure. One constraint can be adjusted through its amplitude. To fix a second constraint, a convolution with an unshaped function would be required (refer to 2.3).

\section{Simulation results}

Consider the system of the Figure 2. This section presents a comparison between different techniques regarding the acceleration-switch condition of the generated input $y(t)$ and the amplitude of the system response $x(t)$. The following are the specifications of the simulations performed.

1- Techniques compared by simulation: Within the methods described in Section 1, some of the commonly used that give null residual vibration for damped systems were chosen to compare with the current proposal: i) Positive ZV shaper ${ }^{6}$, ii) Negative ZV shaper with positive and negative impulses ${ }^{10}(\mathrm{P}=1)$ and iii) the OAT filtering algorithm ${ }^{19}$, with a time-delay of $T=0.2 T_{0}$. This value was chosen to maintain approximately the duration of the impulse shaper to a similar value than the one obtained by the other compared methods, and to limit the magnitude of the impulses generated around the unit (similar to the other methods as well) which is interesting to avoid high amplitudes of the command input. For the new method, a pulse was used as a base function $u \mathrm{~b}(t)$, and the second procedure in 2.3 was followed.

2- Unshaped functions: Versine, double harmonic (Expressions (26) and (27), respectively) and trapezium functions were used. First two present the advantage of having a minimum $C^{1}$ continuity degree at both ends relative to the rest condition, to provide smooth transitions when convolved with shapers. The second is defined as a fast function (slopes duration $0.04 \mathrm{~s}$ ).

$$
\begin{gathered}
u_{\mathrm{a}}(s)=1-\cos (2 \pi s) \quad s \in[0,1] \\
u_{\mathrm{a}}(s)=[1-\cos (2 \pi s)]-\frac{1}{4}[1-\cos (4 \pi s)] \quad s \in[0,1]
\end{gathered}
$$

3- System: The simulations were performed assuming a natural frequency $f_{0}=1 \mathrm{~Hz}$ and damping ratios of $\zeta=0$ and $\zeta=0.2$ in the system in Figure 2.

4- Command generation criteria: Two criteria were used to generate the command inputs: i) The duration of the unshaped function was fixed between 0.2 and 1.0 seconds to see the relative effect of the method regarding the amplitude of the system response, without the influence of this unshaped function, and ii) The duration of the overall input was fixed between 1.1 and 2.0 seconds to evaluate the performance in terms of system response and acceleration switch condition. In both criteria, the resultant inputs were used as displacement, velocity and acceleration profiles and their integral value between $t_{0}$ and $t_{\mathrm{f}}$ was fixed to the unity at the end of the transient, assuming null initial conditions.

To reduce the graphical extension of this section, the velocity profiles were chosen to explain the metrics used and the results obtained, and the rest are described in several summary tables. 


\subsection{Velocity profiles}

Criterion i): Figure 9 is a graphic sample of the comparison done following the indicated command generation criterion, for a system with a damping ratio of $\zeta=0.2$ and a versine as the unshaped command.

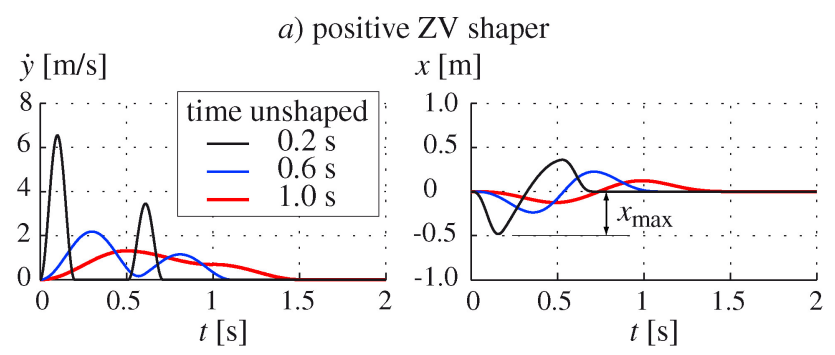

b) negative ZV shaper
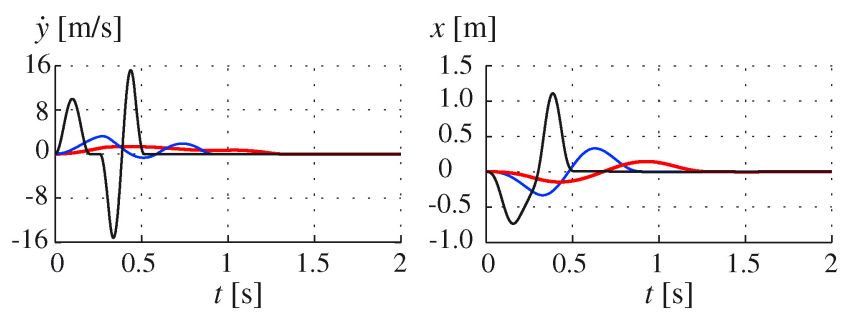

c) OAT filter
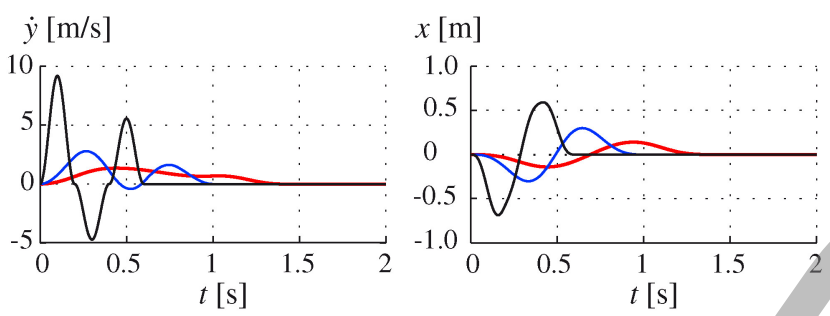

d) proposed method
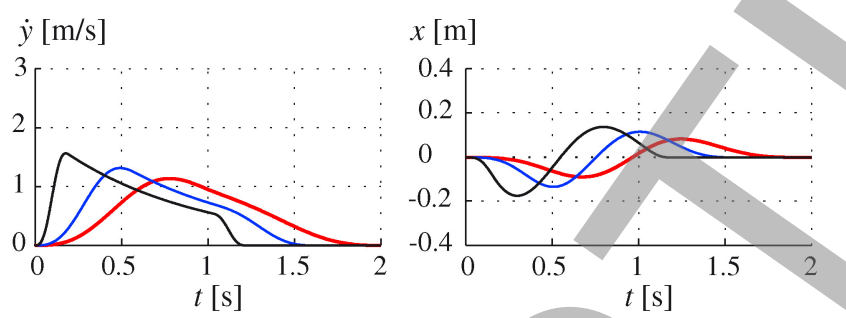

Fig. 9 Velocity inputs and displacement responses for the indicated methods. Criterion i) fixed unshaped function duration

The modulus of the maximum amplitude of the system response during the transient, $\left|x_{\max }\right|$, versus the unshaped command duration is indicated in Figure 10. The proposed technique provides lower maximum amplitudes for all the time range indicated. For $t_{\mathrm{a}}=0.2 \mathrm{~s}$, the maximum amplitudes of the compared methods are in between $0.48 \mathrm{~m}$ and $1.11 \mathrm{~m}$, while it is $0.17 \mathrm{~m}$ for the proposed one. For $t_{\mathrm{a}}=1.0 \mathrm{~s}$, these amplitudes are in between $0.12 \mathrm{~m}$ and $0.15 \mathrm{~m}$, while it is $0.09 \mathrm{~m}$ for the new method.

$\left|x_{\max }\right|[\mathrm{m}]$

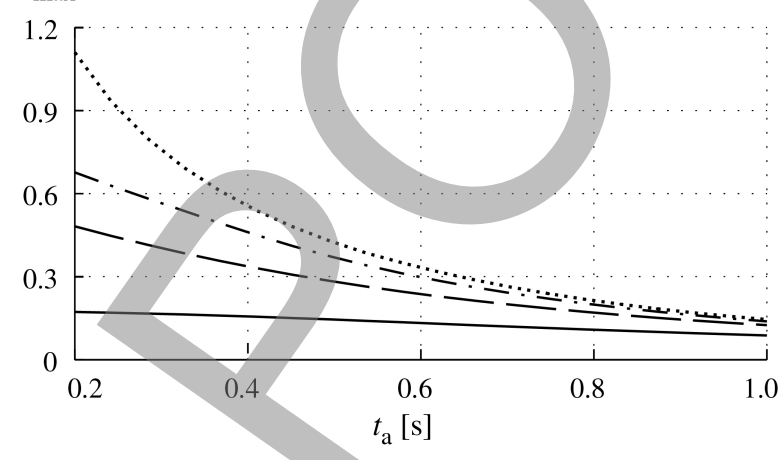

Fig. 10 System response maximum amplitudes versus unshaped command duration: positive ZV shaper (dashed); negative ZV shaper (dotted); OAT filter (dash dotted); proposed method (solid) 

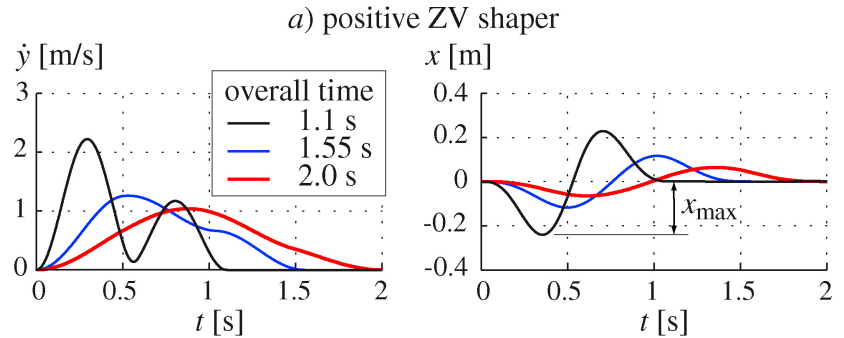

b) negative ZV shaper
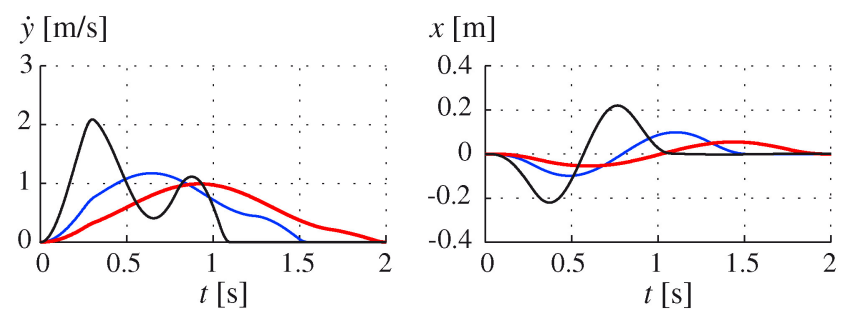

c) OAT filter
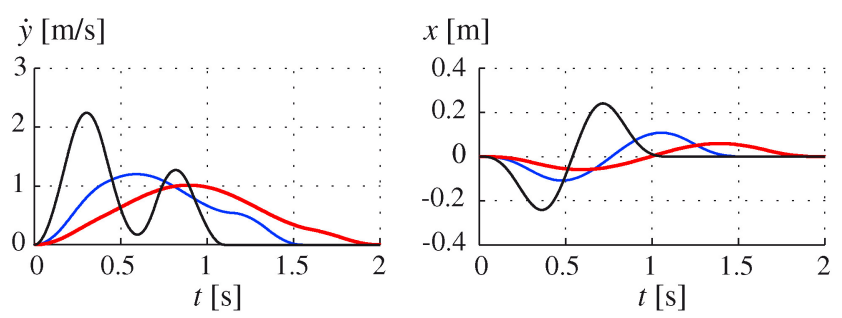

d) proposed method
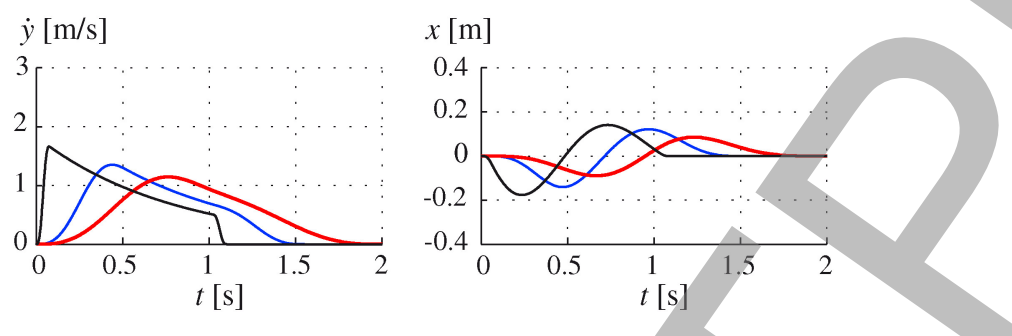

Fig. 11 Velocity inputs and displacement responses for the indicated methods. Criterion ii) fixed overall duration

Criterion ii): Figure 11 is a graphic sample of the comparison done with the indicated criterion. The system has a damping ratio of $\zeta=0.2$ and a versine is used as the unshaped command as well.

The modulus of the maximum amplitude of the system response during the transient, $\left|x_{\max }\right|$, versus the total duration of the input is indicated in Figure 12. In this case, the proposed method provides lower maximum amplitudes up to $1.23 \mathrm{~s}$ or $1.33 \mathrm{~s}$ of total duration, depending on the method compared. As shown, for an input duration of $1.1 \mathrm{~s}$, the maximum amplitudes of the compared techniques are in between $0.22 \mathrm{~m}$ and $0.24 \mathrm{~m}$, while it is $0.18 \mathrm{~m}$ for the proposed one.

Although the acceleration motion profiles are not displayed, the number of alternations between positive and negative values can be deduced easily in Figure 11. The positive and negative ZV shapers and the OAT filter present three sign changes for shorter durations, while a sign change occurs once with the proposed method.

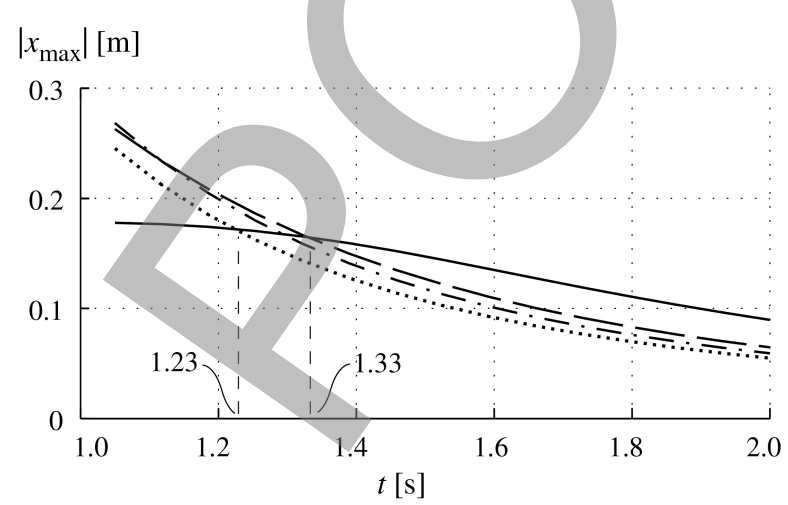

Fig. 12 System response maximum amplitudes versus total input duration: positive ZV shaper (dashed); negative ZV shaper (dotted); OAT filter (dash dotted); proposed method (solid) 


\subsection{Summarized results}

Criterion i): Table 1 is a summary table that includes the system response maximum amplitude for $t_{\mathrm{a}}=0.2 \mathrm{~s}$ (unshaped command duration). The lower amplitudes are marked in grey background.

In all the simulations performed, the maximum amplitudes obtained for the current proposal are lower than for other techniques. The tendencies are analogous to the ones showed in Figure 10.

Criterion ii): Three summary tables that include the relevant results of the simulations made when the generated signal was used as a displacement (Table 2), velocity (Table 3) and acceleration input (Table 4). The best results are marked in grey background.

Table 1 Simulation results. System response maximum amplitude [m]

\begin{tabular}{|c|c|c|c|c|c|c|}
\hline$\zeta$ & $\begin{array}{l}\text { Input used } \\
\text { as }\end{array}$ & $\begin{array}{l}\text { Unshaped } \\
\text { command }\end{array}$ & 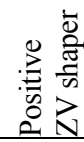 & 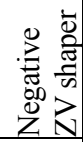 & 崖 & 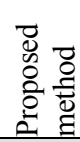 \\
\hline \multirow{9}{*}{0} & \multirow{3}{*}{ Displac. } & Versine & 4.73 & 24.0 & 8.43 & 0.97 \\
\hline & & Double harm. & 6.45 & 31.6 & 10.0 & 0.98 \\
\hline & & Trapezium & 3.11 & 17.2 & 6.6 & 0.95 \\
\hline & \multirow{3}{*}{ Velocity } & Versine & 0.43 & 1.31 & 0.62 & 0.16 \\
\hline & & Double harm. & 0.45 & 1.58 & 0.65 & 0.16 \\
\hline & & Trapezium & 0.40 & 0.92 & 0.6 & 0.15 \\
\hline & \multirow{3}{*}{ Acceleration } & Versine & 0.08 & 0.13 & 0.10 & 0.05 \\
\hline & & Double harm. & 0.08 & 0.14 & 0.10 & 0.05 \\
\hline & & Trapezium & 0.08 & 0.12 & 0.10 & 0.05 \\
\hline \multirow{9}{*}{0.2} & \multirow{3}{*}{ Displac. } & Versine & 5.55 & 18.1 & 8.57 & 1.18 \\
\hline & & Double harm. & 7.76 & 25.5 & 10.9 & 1.29 \\
\hline & & Trapezium & 3.74 & 16.4 & 7.27 & 1.03 \\
\hline & \multirow{3}{*}{ Velocity } & Versine & 0.48 & 1.11 & 0.68 & 0.17 \\
\hline & & Double harm. & 0.52 & 1.39 & 0.73 & 0.17 \\
\hline & & Trapezium & 0.43 & 0.74 & 0.61 & 0.17 \\
\hline & \multirow{3}{*}{ Acceleration } & Versine & 0.08 & 0.11 & 0.10 & 0.05 \\
\hline & & Double harm. & 0.08 & 0.12 & 0.10 & 0.05 \\
\hline & & Trapezium & 0.08 & 0.11 & 0.10 & 0.05 \\
\hline
\end{tabular}

Table 2 Simulation results for displacement profiles

\begin{tabular}{|c|c|c|c|c|c|c|}
\hline$\zeta$ & Metric & $\begin{array}{l}\text { Unshaped } \\
\text { command }\end{array}$ & 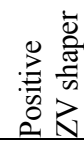 & 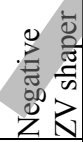 & 还 & $\begin{array}{r}\vec{d} \\
0 \\
0 \\
0 \\
0 \\
0 \\
0 \\
1\end{array}$ \\
\hline \multirow{9}{*}{0} & \multirow{3}{*}{$\begin{array}{l}\text { Acceleration } \\
\text { \# sign } \\
\text { changes }\end{array}$} & Versine & 4 & 4 & 4 & 2 \\
\hline & & Double harm. & 4 & 4 & 4 & 2 \\
\hline & & Trapezium & 6 & 6 & 6 & 2 \\
\hline & \multirow{3}{*}{$x_{\max }[\mathrm{m}]$} & Versine & 2.26 & 1.68 & 2.16 & 0.99 \\
\hline & & Double harm. & 2.70 & 3.75 & 3.82 & 1.00 \\
\hline & & Trapezium & 0.88 & 2.58 & 1.09 & 0.99 \\
\hline & \multirow{3}{*}{$\begin{array}{l}\text { Proposed } \\
\text { method has } \\
\text { lower } x_{\max } \\
\text { up to \# [s] }\end{array}$} & Versine & & & & 1.29 \\
\hline & & Double harm. & & & & 1.74 \\
\hline & & Trapezium & & & & - \\
\hline \multirow{9}{*}{0.2} & \multirow{3}{*}{$\begin{array}{l}\text { Acceleration } \\
\text { \# sign } \\
\text { changes }\end{array}$} & Versine & 6 & 6 & 6 & 4 \\
\hline & & Double harm. & 4 & 4 & 4 & 4 \\
\hline & & Trapezium & 6 & 6 & 6 & 4 \\
\hline & \multirow{3}{*}{$x_{\max }[\mathrm{m}]$} & Versine & 2.25 & 1.76 & 2.16 & 1.50 \\
\hline & & Double harm. & 2.72 & 3.68 & 3.86 & 1.55 \\
\hline & & Trapezium & 1.13 & 2.87 & 1.32 & 1.46 \\
\hline & \multirow{3}{*}{$\begin{array}{l}\text { Proposed } \\
\text { method has } \\
\text { lower } x_{\max } \\
\text { up to \# [s] }\end{array}$} & Versine & & & & 1.24 \\
\hline & & Double harm. & & & & 1.75 \\
\hline & & Trapezium & & & & - \\
\hline
\end{tabular}

The proposed method shows an optimum number of alternations between positive and negative values of acceleration except for the damped displacement profiles where shows the minimum relative to other methods, but not the optimal. The positive ZV shaper also gives the optimal number of sign changes for the acceleration profiles. 
Regarding the amplitude of the system response during the transient, the proposed technique shows the minimum values, when the versine and double harmonic are used, while the positive ZV shaper presents them as well, when the trapezium is used.

Among the simulated unshaped commands, the double harmonic shows the most severe condition regarding the amplitude of the system response during the transient, while the trapezium has the better performance.

Table 3 Simulation results for velocity profiles

\begin{tabular}{|c|c|c|c|c|c|c|}
\hline$\zeta$ & Metric & $\begin{array}{l}\text { Unshaped } \\
\text { command }\end{array}$ & 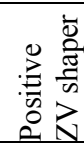 & 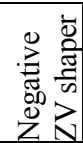 & 岕 & 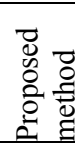 \\
\hline \multirow{9}{*}{0} & \multirow{3}{*}{$\begin{array}{l}\text { Acceleration } \\
\text { \# sign } \\
\text { changes }\end{array}$} & Versine & 3 & 3 & 3 & 1 \\
\hline & & Double harm. & 3 & 3 & 3 & 1 \\
\hline & & Trapezium & 1 & 5 & 5 & 1 \\
\hline & \multirow{3}{*}{$x_{\max }[\mathrm{m}] \cdot 10^{-1}$} & Versine & 2.35 & 2.16 & 2.41 & 1.58 \\
\hline & & Double harm. & 2.94 & 3.37 & 3.37 & 1.59 \\
\hline & & Trapezium & 1.42 & 1.94 & 1.63 & 1.58 \\
\hline & \multirow{3}{*}{$\begin{array}{l}\text { Proposed } \\
\text { method has } \\
\text { lower } x_{\max } \\
\text { up to \# [s] }\end{array}$} & Versine & & & & 1.29 \\
\hline & & Double harm. & & & & 1.68 \\
\hline & & Trapezium & & & & - \\
\hline \multirow{9}{*}{0.2} & \multirow{3}{*}{$\begin{array}{l}\text { Acceleration } \\
\text { \# sign } \\
\text { changes }\end{array}$} & Versine & 3 & 3 & 3 & 1 \\
\hline & & Double harm. & 3 & 3 & 3 & 1 \\
\hline & & Trapezium & 1 & 5 & 5 & 1 \\
\hline & \multirow{3}{*}{$x_{\max }[\mathrm{m}] \cdot 10^{-1}$} & Versine & 2.41 & 2.20 & 2.42 & 1.77 \\
\hline & & Double harm. & 3.08 & 3.32 & 3.42 & 1.77 \\
\hline & & Trapezium & 1.44 & 1.64 & 1.64 & 1.77 \\
\hline & \multirow{3}{*}{$\begin{array}{l}\text { Proposed } \\
\text { method has } \\
\text { lower } x_{\max } \\
\text { up to \# [s] }\end{array}$} & Versine & & & & 1.23 \\
\hline & & Double harm. & & & & 1.60 \\
\hline & & Trapezium & & & & - \\
\hline
\end{tabular}

Table 4 Simulation results for acceleration profiles

\begin{tabular}{|c|c|c|c|c|c|c|}
\hline$\zeta$ & Metric & $\begin{array}{l}\text { Unshaped } \\
\text { command }\end{array}$ & : & 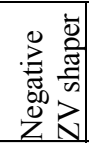 & 岩 & 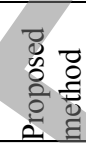 \\
\hline \multirow{9}{*}{0} & \multirow{3}{*}{$\begin{array}{l}\text { Acceleration } \\
\text { \# sign } \\
\text { changes }\end{array}$} & Versine & 0 & 0 & 0 & 0 \\
\hline & & Double harm. & 0 & 2 & 2 & 0 \\
\hline & & Trapezium & 0 & 2 & 0 & 0 \\
\hline & \multirow{3}{*}{$x_{\max }[\mathrm{m}] \cdot 10^{-2}$} & Versine & 6.28 & 5.92 & 6.29 & 5.04 \\
\hline & & Double harm. & 6.89 & 7.48 & 7.48 & 5.05 \\
\hline & & Trapezium & 4.52 & 3.31 & 3.84 & 5.03 \\
\hline & \multirow{3}{*}{$\begin{array}{l}\text { Proposed } \\
\text { method has } \\
\text { lower } x_{\max } \\
\text { up to \# [s] }\end{array}$} & Versine & & & & 1.29 \\
\hline & & Double harm. & & & & 1.72 \\
\hline & & Trapezium & & & & - \\
\hline \multirow{9}{*}{0.2} & \multirow{3}{*}{$\begin{array}{l}\text { Acceleration } \\
\text { \# sign } \\
\text { changes }\end{array}$} & Versine & 0 & 0 & 0 & 0 \\
\hline & & Double harm. & 0 & 2 & 2 & 0 \\
\hline & & Trapezium & 0 & 2 & 0 & 0 \\
\hline & \multirow{3}{*}{$x_{\max }[\mathrm{m}] \cdot 10^{-2}$} & Versine & 6.28 & 5.99 & 6.30 & 5.02 \\
\hline & & Double harm. & 6.86 & 7.42 & 7.49 & 5.03 \\
\hline & & Trapezium & 4.61 & 3.42 & 3.84 & 5.02 \\
\hline & \multirow{3}{*}{$\begin{array}{l}\text { Proposed } \\
\text { method has } \\
\text { lower } x_{\max } \\
\text { un to } \#[\mathrm{~s}]\end{array}$} & Versine & & & & 1.30 \\
\hline & & Double harm. & & & & 1.73 \\
\hline & & Trapezium & & & & - \\
\hline
\end{tabular}

\subsection{Limitations}

The proposed method obtains command inputs with a minimum-acceleration-switch condition and limited amplitude maximum values with good efficiency in vibration reduction. Four known limitations are described compared with the positive ZV method:

1- Minimum command total duration: For single-mode systems, the minimum command total duration with the current proposal is $1 / f_{\mathrm{d}}$ when using a rectangular pulse as $u_{\mathrm{b}}(t)$. This limit is $1 /\left(2 f_{\mathrm{d}}\right)$ for the positive ZV shaper.

2- Velocity and distance possible values when an acceleration command $\ddot{y}(t)$ is used: The velocity and the distance achieved during the transient 
are two of the possible functional requirements to be fixed when an acceleration command is used. However, not all the values desired can be obtained. To evaluate these limits, an ideal case was analyzed by using an impulse as unshaped command $u_{\mathrm{a}}(t)$ to be convolved with the correspondent shaper. The proposed method is compared with the positive ZV shaper in a single-mode system. Assume also that $\ddot{y}(t)$ is defined between $t_{0}=0$ and $t_{\mathrm{f}}$ with null velocity and displacement initial conditions, i.e., $y\left(t_{0}\right)=0$ and $\dot{y}\left(t_{0}\right)=0$. For the positive ZV shaper, the acceleration command is given by

$$
\begin{aligned}
& \ddot{y}(t)=A \delta(t) \quad t=0 \\
& \ddot{y}(t)=A \delta(t) \mathrm{e}^{-\zeta 2 \pi f_{0} \frac{1}{2 f_{\mathrm{d}}}} \quad t=1 /\left(2 f_{\mathrm{d}}\right) \\
& \ddot{y}(t)=0 \quad \forall t \neq\left\{\begin{array}{l}
0 \\
1 /\left(2 f_{\mathrm{d}}\right)
\end{array}\right.
\end{aligned}
$$

where $\delta(t)$ describes a unitary impulse. The velocity at $t_{\mathrm{f}}=1 /\left(2 f_{\mathrm{d}}\right)^{+}(+$means just after the impulse $)$is given by

$$
\dot{y}\left(t_{\mathrm{f}}\right)=A\left(1+\mathrm{e}^{-\zeta 2 \pi f_{0} / 2 f_{\mathrm{d}}}\right)
$$

and displacement at $t_{\mathrm{f}}$ is

$$
y\left(t_{\mathrm{f}}\right)=\frac{A}{2 f_{\mathrm{d}}}
$$

By modifying the parameter $A$, different values of velocity and displacement are obtained. The relation between them is given by the Expression (31) and is the line indicated in Figure $13(a)$.

$$
\dot{y}\left(t_{\mathrm{f}}\right)=2 f_{0} \sqrt{1-\zeta^{2}}\left(1+\mathrm{e}^{-\pi \zeta / \sqrt{1-\zeta^{2}}}\right) y\left(t_{\mathrm{f}}\right)
$$

If the positive ZV shaper is now convolved with a feasible unshaped function, by modifying the total command amplitude and the unshaped function duration, the user will be able to choose two independent values of velocity and distance within the grey area of Figure $13(a)$.

If the proposed method is used, assuming a rectangular pulse as $u_{\mathrm{b}}(t)$, the acceleration command can be described by

$$
\begin{aligned}
& \ddot{y}(t)=A \mathrm{e}^{-\zeta 2 \pi f_{0} t} \quad 0 \leq t<1 / f_{\mathrm{d}} \\
& \ddot{y}(t)=0 \quad\left\{\begin{array}{l}
t<0 \\
t \geq 1 / f_{\mathrm{d}}
\end{array}\right.
\end{aligned}
$$

The velocity at $t_{\mathrm{f}}=1 / f_{\mathrm{d}}$ is given by

The displacement at $t_{\mathrm{f}}$ is

$$
\dot{y}\left(t_{\mathrm{f}}\right)=\frac{A}{\zeta 2 \pi f_{0}}\left(1-e^{-\zeta 2 \pi f_{0} / f_{d}}\right)
$$

$$
y\left(t_{\mathrm{f}}\right)=\frac{A}{\zeta 2 \pi f_{0}}\left(\frac{1}{f_{\mathrm{d}}}-\frac{1}{\zeta 2 \pi f_{0}}+\frac{1}{\zeta 2 \pi f_{0}} e^{-\zeta 2 \pi f_{0} / f_{d}}\right)
$$

As stated before, by modifying the parameter $A$ different values of velocity and displacement are obtained. The relation between them is given by the expression (35) and is the line indicated in Figure $13(b)$.

$$
\dot{y}\left(t_{\mathrm{f}}\right)=\frac{\zeta 2 \pi f_{0} \sqrt{1-\zeta^{2}}\left(\mathrm{e}^{2 \pi \zeta / \sqrt{1-\zeta^{2}}}-1\right)}{\left(2 \pi \zeta-\sqrt{1-\zeta^{2}}\right) \mathrm{e}^{2 \pi \zeta / \sqrt{1-\zeta^{2}}}+\sqrt{1-\zeta^{2}}} y\left(t_{\mathrm{f}}\right)
$$

If the proposed method is now convolved with a feasible unshaped function, following a similar reasoning to that of the former method, the user will be able to choose two independent values of velocity and distance within the grey area of Figure $13(b)$, which is smaller than for the positive ZV shaper. 

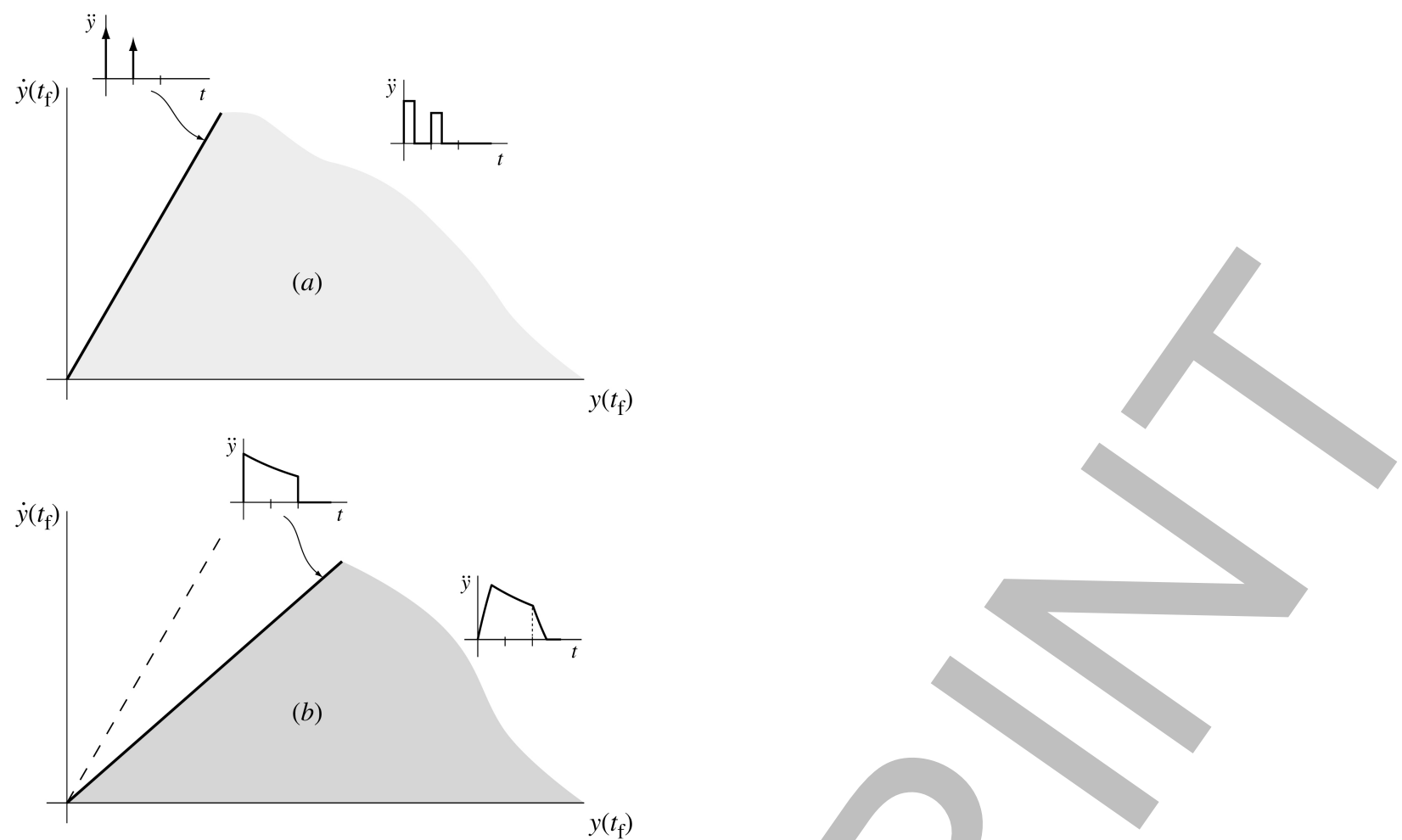

Fig. 13 Comparative space of velocity-distance solutions between positive ZV shaper $(a)$ and the proposed method $(b)$

3- Maximum command acceleration when a velocity command $\dot{y}(t)$ is used: With the proposed method, higher initial command accelerations can be observed when the generated signal is used as a velocity command $\dot{y}(t)$. However, the forces required from the actuator are not necessarily higher than other methods, because the command input does not directly describe the acceleration of the inertia of the system. In fact, the amplitude of the system response for the proposed method is lower in several cases, compared to the other techniques, as shown in Figure 11.

4- Computation complexity: The algorithm used by the proposed method requires more computation complexity compared to the ZV shaper, because it includes a convolution operation. By using a $3 \mathrm{GHz}$ processor, a sampling frequency of $1 \mathrm{kHz}$, and considering that the profile has an overall duration of $2 \mathrm{~s}$, the computing time for the proposed method is around $10 \%$ higher.

\section{Experimental results}

A carriage-pendulum test bed was built to check the goodness of the method proposed. The system has two degrees of freedom: one defined by the pendulum rotation, which has a free oscillatory behavior, and the other defined by the carriage movement, which is forced with the desired motion law. As shown in Figures 14 and 15, the pendulum of mass $m$, inertia $I$ and length $l$ was assembled on a carriage through a commercial rotary damper, which provided the required damping $c$. The carriage displacement is defined by the coordinate $y$ and follows a command input driven with a PI control, a DC motor and a belt-pulley transmission. The feedback for this control is provided by an incremental encoder, which measures the carriage displacement directly. The angular coordinate of the pendulum is defined by $\theta$, and its angular velocity is measured by means of a gyroscope integrated circuit assembled on the rod near the pendulum rotary axis. The table 4 shows a summary of the values and devices used for this test bed.

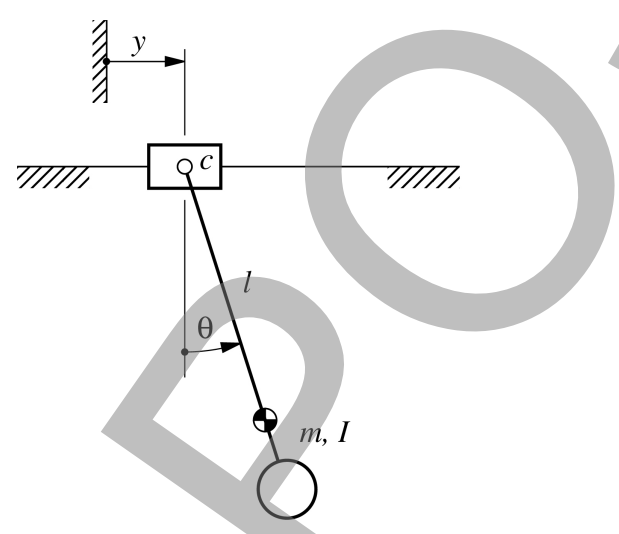

Fig. 14 Carriage-pendulum test bed schematics and coordinates 


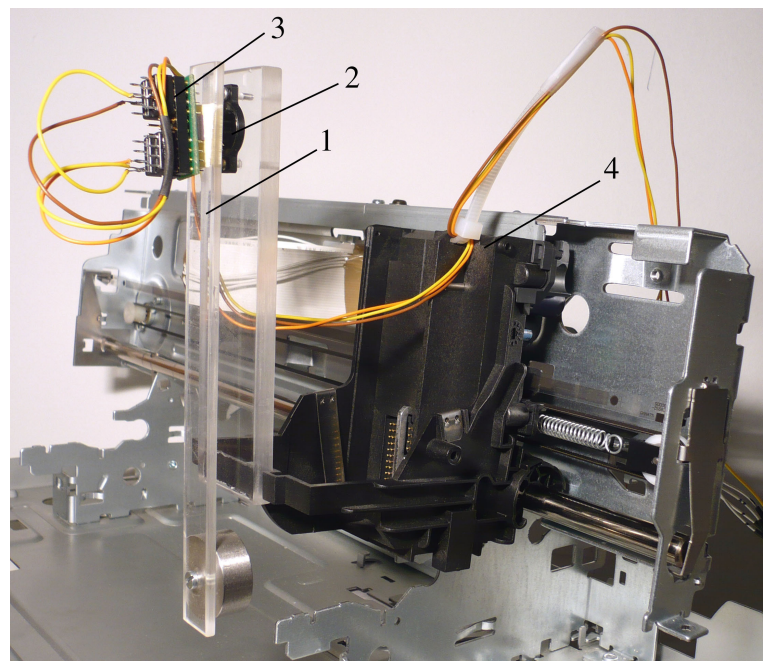

Fig. 15 Carriage-pendulum test bed: 1) pendulum, 2) rotary damper, 3) gyroscope, 4) carriage

The motion equation of this pendulum test bed is described by

$$
\left(m l^{2}+I\right) \ddot{\theta}+c \dot{\theta}+m \mathrm{~g} l \sin \theta=-m \ddot{y}(t) l \cos \theta
$$

Although it is a non-linear system, this type of mechanism was chosen because of its simplicity. To minimize the non-linear effects, the inputs were adjusted to work in a small angular range. In this case, angular nonlinearity effects can be neglected below a range of $\theta= \pm 20^{\circ}$. The rotary damper assembled on this mechanism can be identified as another source of non-linearity, according to the information delivered from the supplier. However, by using it in a low angular velocity range there is a small effect on the overall results, so an ideal linear damping $c$ can be assumed. By linearizing the motion equation around $\theta=0$ assuming small oscillations during and after the transient around this position, the motion equation can be described by Expression (37) which follows the Expression (1) of the system in Figure 2.

$$
\left(m l^{2}+I\right) \ddot{\theta}+c \dot{\theta}+m \mathrm{~g} l \theta=-m \ddot{y}(t) l
$$

Table 4 Test bed parameters and specifications

\begin{tabular}{|l|l|}
\hline Parameter / device & Value / specification \\
\hline$m$ & $36.20 \cdot 10^{-3} \mathrm{~kg}$ \\
\hline$I$ & $2.82 \cdot 10^{-6} \mathrm{~kg} \cdot \mathrm{m}^{2}$ \\
\hline$l$ & $110 \mathrm{~mm}$ \\
\hline$c$ & $1.30 \cdot 10^{-3} \mathrm{Nm} /(\mathrm{rad} / \mathrm{s})$ \\
\hline Motor & Permanent magnet DC brushed \\
\hline Encoder & Linear, 150 lines $/$ inch $2 \mathrm{ch}$ output \\
\hline Gyroscope & ADXRS300, $\pm 300 \%$, $5 \mathrm{mV} /(\% / \mathrm{s})$ \\
\hline Power supply & DC $15 \mathrm{~V}, 4 \mathrm{~A}$ \\
\hline Power electronics & Galil Motion Control MSA12-80 \\
\hline Control electronics & NI PCI-6036E \\
\hline Control software & Matlab Simulink $®$ \\
\hline
\end{tabular}

The test-bed setup gives a measured oscillation frequency of $f_{\mathrm{d}}=1.42 \mathrm{~Hz}$ and a damping ratio of $\zeta=0.15$. Therefore, the system natural frequency obtained by calculation is $f_{0}=1.44 \mathrm{~Hz}$. The bandwidths of the motor and the electronics should cover the natural frequency of the system with an adequate margin to avoid the filtering effect on the command input around this frequency. The motor-carriage inertia used in this application shows a measured frequency bandwidth of $14 \mathrm{~Hz}$, and the electronics gives a frequency bandwidth of $2.5 \mathrm{kHz}$, according to the supplier specifications. Thus, both devices have values far from the system resonance.

Two sets of tests were performed. The first one compares the benefits of including in the desired input the product of the base function with a negative exponential time function (which takes into account the system damping ratio), relative to the use of the base function alone. The second compares the proposed method with the positive and negative ZV shapers and the OAT filter. In both examples, point-to-point movements described through velocity command $\dot{y}(t)$ were carried out.

The first test compares two command inputs $\dot{y}(t)$ (Figure 16) with the transient time fixed at 0.9 seconds and the distance fixed at $220 \mathrm{~mm}$. The first one is a trapezoidal input designed by convolving a pulse of $1 / f_{\mathrm{d}}$ duration with a pulse of $0.9-1 / f_{\mathrm{d}}$ and omitting the system damping ratio $(\zeta=0)$. It is given by

$$
\dot{y}_{1}(t)=A_{1}\left[u_{\mathrm{b}}(t) \otimes u_{\mathrm{a}}(t)\right]
$$

The design of the second motion law includes the system damping ratio $\zeta$ in the former case and is described by 


$$
\dot{y}_{2}(t)=A_{2}\left[u_{\mathrm{b}}(t) \otimes u_{\mathrm{a}}(t)\right] \mathrm{e}^{-\zeta 2 \pi f_{0} t}
$$

The constants $A_{1}$ and $A_{2}$ were calculated to handle a $220 \mathrm{~mm}$ distance. The displacement profiles are also shown in the figure. A relevant difference in terms of residual vibration can be observed in the system output $\theta(t)$. While the amplitude of the oscillation during the transient is higher for the motion law defined with the proposed method, the result in terms of residual vibrations is almost zero in this case.

The system output $\theta(t)$ appears to be free from measurement noise because of the integration of the measured angular velocity $\dot{\theta}(t)$ from the gyroscope, which is equivalent to a low pass filtering process. The delay between the beginning of the input and the beginning of the output is considered to be normal and is caused by the integral effect of the PI control system.
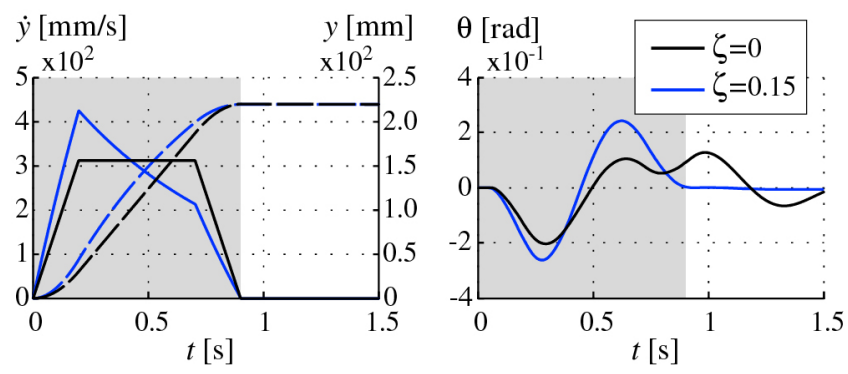

Fig. 16 Experimental results of the proposed method: command inputs (with associated displacements in dashed lines) and system responses. Residual vibration when the damping ratio is omitted

The results of the second test are presented in Figure 17. Four command inputs $\dot{y}(t)$ designed with the indicated techniques are compared. The transient time was fixed to $1.1 / f_{0}=0.76 \mathrm{~s}$, the distance was fixed at $160 \mathrm{~mm}$ and a versine was used as unshaped command. For the proposed method, the input designed is described by

$$
\dot{y}_{2}(t)=A_{2}\left[u_{\mathrm{b}}(t) \mathrm{e}^{-\zeta 2 \pi f_{0} t}\right] \otimes u_{\mathrm{a}}(t)
$$

In terms of residual vibration, no significant differences are revealed in the output signals $\theta(t)$, which is almost zero in all the cases. With regard to the maximum amplitude of the system response, the positive and negative ZV shapers, and the OAT filter present $38 \%, 29 \%$ and $43 \%$ higher amplitudes than the proposed method, respectively. Finally, the number of sign changes of the acceleration profiles follows what was expected by simulation. 

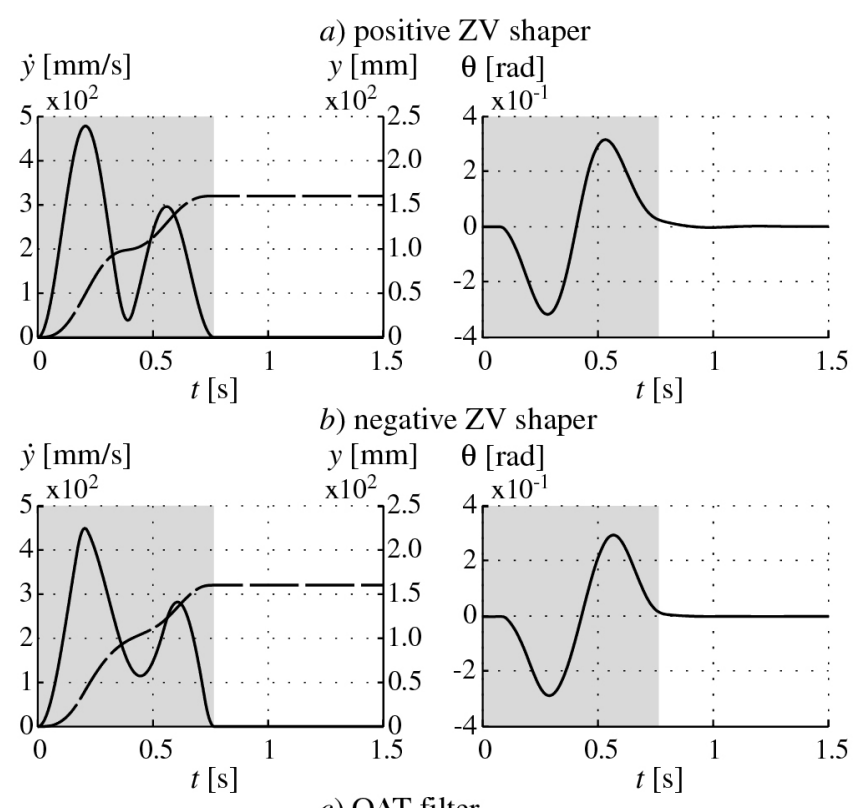

c) OAT filter
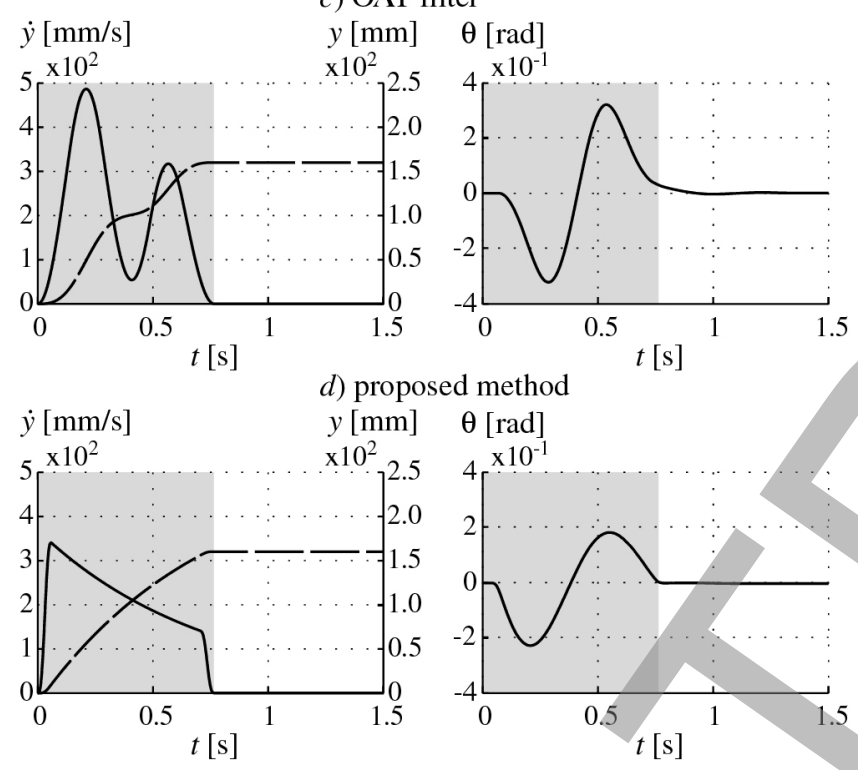

Fig. 17 Experimental results: Velocity inputs (with associated displacements in dashed line) and system responses for the indicated methods

\section{Conclusions}

A time-domain method to design command inputs to reduce residual vibration in damped linear systems was developed. The technique is based on the generation of input commands with zero-crossing points in their frequency spectra at the system resonances and is extended to damped systems by including an appropriate negative exponential time function related to the damping ratio and the natural frequency of the system. By convolving this command input with any unshaped one, a minimum of two functional requirements or constraints can be fixed. This approach is extended to multiple-mode systems, any of them characterized by their oscillation frequency and damping ratio. In this case, the final input is obtained by convolving each of the individual inputs designed for each system mode. Compared to other ZV methods, for short duration input range the signals obtained have better performance in terms of minimum-acceleration-switch condition, which can be translated to smoother shapes of the motion profiles. Finally, a damped pendulum test bed was used to conduct some experiments, confirming the effectiveness and usefulness of the method.

\section{REFERENCES}

1. Singhose, W.E., "Command Shaping for Flexible Systems: A Review of the First 50 Years", International Journal of Precision Engineering and Manufacturing, Vol. 10, No. 4, pp. 153-168, 2009.

2. Singhose, W.E., Seering W., “Command Generation For Dynamic Systems”, Singhose, W.E. (www.lulu.com), 2011.

3. Aspinwall, D.M., "Acceleration Profiles for Minimizing Residual Response”, ASME Journal of Dynamic Systems, Measurement, and Control, Vol. 102, No. 3, pp. 3-6, 1980.

4. Meckl, P.H., Seering, W.P., "Minimizing Residual Vibration for Point-to-Point Motion”, ASME Journal of Vibration, Acoustics, Stress, and Reliability in Design, Vol. 107, No. 3, pp. 378-382, 1985. 
5. Meckl, P.H., Seering, W.P., "Experimental Evaluation of Shaped Inputs to Reduce Vibration for a Cartesian Robot," ASME Journal of Dynamic Systems, Measurement, and Control, Vol. 112, No. 2, pp. 159-165, 1990.

6. Singer, N.C., Seering, W.P., "Preshaping Command Inputs to Reduce System Vibration", ASME Journal of Dynamic Systems, Measurement, and Control, Vol. 112, No. 1, pp. 76-82, 1990.

7. Hyde, J.M., Seering, W.P., "Using Input Command Pre-Shaping to Suppress Multiple Mode Vibration”, IEEE Proceedings of the International Conference on Robotics and Automation, pp. 2604-2609, 1991.

8. Singhose, W.E., Seering, W.P., Singer, N.C., "Residual Vibration Reduction Using Vector Diagrams to Generate Shaped Inputs", ASME Journal of Mechanical Design, Vol. 116, pp. 654-659, 1994.

9. Gürleyük, S.S., Cinal, S., "Robust Three-impulse Sequence Input Shaper Design”, Journal of Vibration and Control, Vol. 13, No. 12, pp. 1807-1818, 2007.

10. Singhose, W.E., Seering, W.P., Singer, N.C., "Time-Optimal Negative Input Shapers”, ASME Journal of Dynamic Systems, Measurement, and Control, Vol. 119, No. 2, pp. 198-205, 1997.

11. Chang, T., Godbole, K., Hou, E., “Optimal Input Shaper Design For High-Speed Robotic Workcells”, Journal of Vibration and Control, Vol. 9, pp. 1359-1376, 2003.

12. Singh, T., Heppler, G.R., "Shaped Input Control of a System With Multiple Modes", ASME Journal of Dynamic Systems, Measurement, and Control, Vol. 115, No. 3, pp. 341-347, 1993.

13. Singhose, W., Banerjee, A., Seering, W., "Slewing Flexible Spacecraft with Deflection-Limiting Input Shaping", AIAA Journal of Guidance, Control, and Dynamics, vol. 20, No. 2, pp. 291-298, 1997.

14. Kojima, H., Singhose, W., "Adaptive Deflection Limiting Control for Slewing Flexible Space Structures", AIAA Journal of Guidance, Control, and Dynamics, vol. 30, No. 1, pp. 61-67, 2007.

15. Singer, N.C., Singhose, W.E., Seering, W.P., “Comparison of Filtering Methods for Reducing Residual Vibration”, European Journal of Control, Vol. 5, No. 2-4, pp. 208-218, 1999.

16. Meckl, P.H., Seering, W.P., "Reducing Residual Vibration in Systems with Time-Varying Resonances", IEEE International Conference on Robotics and Automation, pp. 1690-1695, 1987.

17. Bhat, S.P., Miu, D.K., "Precise Point-to-Point Positioning Control of Flexible Structures", ASME Journal of Dynamic Systems, Measurement, and Control, Vol. 112, No. 4, pp. 667-674, 1990.

18. Tuttle, T.D., Seering, W.P., “A Zero-placement Technique for Designing Shaped Inputs to Suppress Multiple-mode Vibration”, Proceedings of the American Control Conference, pp. 2533-2537, 1994.

19. Magee, D.P., Book, W.J., "Optimal Filtering to Minimize the Elastic Behavior in Serial Link Manipulators”, Proceedings of the American Control Conference, pp. 2637-2642, 1998.

20. Starr, G. P. "Swing-Free Transport of Suspended Objects with a Path-Controlled Robot Manipulator," ASME Journal of Dynamic Systems, Measurement, and Control, Vol. 107, No. 1, pp. 97-100, 1985.

21. Sorensen, K., Singhose, W., Dickerson, S., "A Controller Enabling Precise Positioning and Sway Reduction in Bridge and Gantry Cranes," Control Engineering Practice, Vol. 15, No. 7, pp. 825-837, 2007.

22. Kim, D., Singhose, W., "Performance Studies of Human Operators Driving Double-Pendulum Bridge Cranes," Control Engineering Practice, Vol. 18, No. 6, pp. 567-576, 2010.

23. Stergiopoulos, J., Konstantopoulos, G., Tzes, A., "Experimental Verification of an Adaptive Input Shaping Scheme for Hoisting Cranes," Mediterranean Conference on Control and Automation, pp. 730-735, 2009.

24. Bhat, S.P., Tanaka, M., Miu, D.K. "Experiments on Point-to-Point Position Control of a flexible Beam Using Laplace Transform TechniquePart 1: Open-Loop", ASME Journal of Dynamic Systems, Measurement, and Control, Vol. 113, pp. 432-437, 1991.

25. Hyde, J.M., Seering, W.P., "Inhibiting Multiple Mode Vibration in Controlled Flexible Systems," Proceedings of the American Control Conference, pp. 2449-2454, 1991. 